\title{
BMJ Open Consequences of health condition labelling: protocol for a systematic scoping review
}

\author{
Rebecca Sims (D) , ${ }^{1}$ Luise Kazda (D) , ${ }^{2}$ Zoe A Michaleff (D) , ${ }^{1}$ Paul Glasziou (D) , \\ Rae Thomas (i) ${ }^{1}$
}

To cite: Sims R,

Kazda L, Michaleff ZA, et al. Consequences of health condition labelling: protocol for a systematic scoping review. BMJ Open 2020;10:e037392. doi:10.1136/ bmjopen-2020-037392

- Prepublication history and additional material for this paper is available online. To view these files, please visit the journal online (http://dx.doi.org/10. 1136/bmjopen-2020-037392).

Received 31 January 2020 Revised 24 August 2020 Accepted 22 September 2020

Check for updates

(c) Author(s) (or their employer(s)) 2020. Re-use permitted under CC BY-NC. No commercial re-use. See rights and permissions. Published by BMJ.

${ }^{1}$ Institute for Evidence-Based Healthcare, Bond University Faculty of Health Sciences and Medicine, Gold Coast, Queensland, Australia

${ }^{2}$ Sydney School of Public Health, The University of Sydney, Sydney, New South Wales, Australia

Correspondence to

Dr Rae Thomas;

rthomas@bond.edu.au

\section{ABSTRACT}

Introduction When health conditions are labelled it is often to classify and communicate a set of symptoms. While diagnostic labelling can provide explanation for an individual's symptoms, it can also impact how individuals and others view those symptoms. Despite existing research regarding the effects of labelling health conditions, a synthesis of these effects has not occurred. We will conduct a systematic scoping review to synthesise the reported consequences and impact of being given a label for a health condition from an individual, societal and health practitioner perspective and explore in what context labelling of health conditions is considered important. Methods and analysis The review will adhere to the Joanna Briggs Methodology for Scoping Reviews. Searches will be conducted in five electronic databases (PubMed, Embase, PsycINF0, Cochrane, CINAHL). Reference lists of included studies will be screened and forward and backward citation searching of included articles will be conducted. We will include reviews and original studies which describe the consequences for individuals labelled with a non-cancer health condition. We will exclude hypothetical research designs and studies focused on the consequences of labelling cancer conditions, intellectual disabilities and/or social attributes. We will conduct thematic analyses for qualitative data and descriptive or meta-analyses for quantitative data where appropriate.

Ethics and dissemination Ethical approval is not required for a scoping review. Results will be disseminated via publication in a peer-reviewed journal, conference presentations and lay-person summaries on various online platforms. Findings from this systematic scoping review will identify gaps in current understanding of how, when, why and for whom a diagnostic label is important and inform future research.

\section{INTRODUCTION}

The diagnosis of physical and psychological health conditions is increasing in prevalence. ${ }^{1-5}$ Diagnoses often occur in the context of individuals seeking to identify and treat symptoms. However, diagnoses can also occur as a result of screening tests where individuals have no discernible signs or symptoms of disease (such as when a routine test determines an individual has hypertension), ${ }^{6}$
Strengths and limitations of this study

- A broad, comprehensive search strategy will be conducted in five electronic databases.

- We will include both qualitative and quantitative studies which will enhance our current understanding of the consequences of health condition labelling.

- Two reviewers will screen $10 \%$ of titles and abstracts, extract data and assess the quality of included studies.

- Eligibility will not be limited to specific health conditions, therefore, the consequences identified will be generalisable to health condition labelling more broadly.

- Articles will be limited to peer-reviewed publications and not include grey or theory-based literature.

from unanticipated findings in investigations for other health concerns (such as identifying an anomaly in a person's thyroid when conducting an MRI of the spine $)^{7}$ or, when people are newly diagnosed with a health condition because of changes to diagnostic thresholds or cut-offs for the condition opposed to changes in individual circumstances (such as for gestational diabetes). ${ }^{1}$ The value of a diagnosis, particularly in these latter contexts, is not always evident and the risk of overdiagnosis and misdiagnosis is significant. ${ }^{189}$

Diagnostic labels provide healthcare professionals with a framework from which to organise and interpret clinical symptom presentations, support clinical decision making through directing treatment decisions, and provide information on possible condition course and overall prognosis. ${ }^{10} 11$ Further, diagnostic labels allow clinicians to assume homogeneity among members of patient groups, in addition to providing an efficient method for health professionals to communicate. $^{12}$

Despite well-meaning intentions, application of diagnostic labels in real-world practice 
can be problematic. Diagnostic criteria can often be ambiguous. For example, symptoms of anxiety, such as restlessness, fatigue or difficulty concentrating, may be explained by diagnoses of anxiety, depressive, or bipolar and related disorders. ${ }^{13}{ }^{14}$ Similarly, chest pain symptoms may be explained by several alternative diagnostic categories such as inflammatory diseases, musculoskeletal conditions or coronary diseases. ${ }^{1516}$ Lastly, non-specific low back pain is the leading cause of disability worldwide, yet for the majority of people, no pathoanatomical cause can be identified. ${ }^{17}$

From the perspective of a patient, a diagnostic label can have a significant impact (negative and positive) on their health outcomes, psychological well-being and behaviour, and can influence how they are viewed and managed by healthcare professionals and are perceived by other members in society (eg, school, workplace). ${ }^{3518}$ In a cohort of over 33000 adults, individuals who were aware that they had hypertension reported elevated levels of psychological distress compared with those individuals who had hypertension, however, were unaware of this. ${ }^{3}$ A study investigating the impact of labelling borderline personality disorder on clinician interpretation of patient symptoms found clinicians' prior awareness of a diagnosis of borderline personality disorder, compared to no awareness, resulted in a tendency to frame observations of the individual in terms of the label, and a failure to observe positive behaviours. ${ }^{12}$

Conversely, a diagnostic label may have positive effects on the individual. These include timely referral to necessary healthcare which, in turn, can reduce morbidity and mortality, improve predictions regarding condition progression as well as facilitate access to support, services and resources (eg, diagnosis-based school funding ${ }^{19} 20$ and social support ${ }^{5}$ ), and provide an explanation and validation of an individual's signs and symptoms. A recent study exploring the impact of chronic fatigue syndrome using hypothetical scenarios of a close friend's diagnosis reported a label of chronic fatigue, compared with no label, elicited higher sympathetic responses from participants, greater potential social support and greater support for active treatment. ${ }^{5}$

The terms used to describe a diagnostic label have been found to influence an individual's behaviour, psychological well-being and treatment preferences. Specifically, a diagnostic label that uses medicalised and precise terminology compared with a description of symptoms has been found to result in higher patient anxiety, greater perceived severity of the condition and a patient preference of more invasive treatments. ${ }^{18}{ }^{21-23}$ This has been evidenced in conditions including gastro-oesophageal reflux disease, polycystic ovary syndrome, bone fracture and low back pain. ${ }^{18}{ }^{21-23}$ Similarly, research suggests that patients diagnosed with diabetes demonstrate a propensity to medical interventions, including insulin use, oral medication taking and blood glucose monitoring, compared to less invasive interventions, such as changes to diet and exercise practices. ${ }^{24}$ The use of a medicalised label over a descriptive label for a health condition is also suggested to result in increased confidence in the medical professional and greater adoption of sick role behaviour. ${ }^{25}$ Alternatively, use of descriptive labels for health conditions was found to be associated with greater patient ownership of the condition. ${ }^{25}$

To date, our understanding of the consequences and impacts of a diagnostic label has been limited to a single perspective (eg, patient, healthcare practitioner), single condition (eg, gastro-oesophageal reflux disease), or restricted to a specific study design (eg, hypothetical research design) and a comprehensive synthesis of this information across health conditions is lacking. ${ }^{26} 27$ Further, exploring the real-world impact of a diagnostic label including benefits and harms has received little attention. ${ }^{22} 2829$ Therefore, the aims of this systematic scoping review are to systematically review original and synthesised research exploring the consequences of being given a label for a health condition to:

1. Identify the range of potential consequences of labelling of health conditions from an individual, societal and health practitioner viewpoint.

2. Explore why, for whom, and in what contexts labelling of health conditions is, or is not, influential.

3. Evaluate the methods used to study the impact of labelling health conditions.

\section{METHODS AND ANALYSIS}

Scoping reviews are suggested as an alternative to systematic reviews, allowing for a broader examination and synthesis of existing research and identification of research gaps. ${ }^{30}$ The proposed systematic scoping review will adhere to the Joanna Briggs Methodology for Scoping Reviews, ${ }^{31}$ and adhere to the Preferred Reporting Items for Systematic Reviews and Meta-Analyses Extension for Scoping Reviews (PRISMA-ScR). ${ }^{32}$ This approach was selected to allow sufficient documentation of the review process. An initial search was conducted in August 2019 to pilot the screening process and data extraction spreadsheet. The review is expected to be complete by October 2020.

\section{Consumer involvement in scoping review design and framework development}

A convenience sampling survey was conducted to explore the publics opinion of the consequences of a diagnostic label for health conditions. In April 2019, we posted the questions 'What are the labelling consequences of being given a health diagnosis? We're working up a list and so far we have: anxiety, relief, more tests, stigma, medicolegal problems. What else?' on two social media platforms, Facebook and Twitter. Responses on Facebook included 14 comments from 6 individuals, while Twitter responses resulted in 45 comments from 40 individuals. The results of this survey were used to inform the development of the search strategy, inclusion and exclusion criteria, data extraction form and an initial qualitative 
Table 1 Coding framework of social media responses

\begin{tabular}{|c|c|c|}
\hline Name & Description & Examples \\
\hline Psychological impact & Psychological impact of diagnosis & $\begin{array}{l}\text { Increased self-understanding } \\
\text { Stigma (internalised stigma (self); perceived stigma from } \\
\text { others) } \\
\text { Increased psychological distress (anxiety, depression, } \\
\text { phobia, worry, fear, stress) }\end{array}$ \\
\hline Support & $\begin{array}{l}\text { Support gained or lost as a result of } \\
\text { diagnosis }\end{array}$ & $\begin{array}{l}\text { Support groups: increased support of others with a } \\
\text { similar diagnosis; network with other patients } \\
\text { Others less respectful, more withdrawn and judgemental }\end{array}$ \\
\hline \multicolumn{3}{|l|}{ Development } \\
\hline Education & $\begin{array}{l}\text { Seeking to become more informed on } \\
\text { diagnoses, testing, intervention }\end{array}$ & $\begin{array}{l}\text { Increase in health literacy due to motivation to find about } \\
\text { treatment options }\end{array}$ \\
\hline Planning & $\begin{array}{l}\text { Forward planning and decision making } \\
\text { as a result of diagnosis }\end{array}$ & $\begin{array}{l}\text { Ability to plan-even if there may not be treatment, } \\
\text { provides an opportunity to get affairs in order (eg, wills) }\end{array}$ \\
\hline Employment & Effect of diagnosis on employment & More sick days; time off work; absenteeism \\
\hline Financial & Effect of diagnosis on finances & $\begin{array}{l}\text { Diagnosis provides access to funds (eg, Medicare, } \\
\text { National Disability Insurance Scheme (NDIS), insurance) }\end{array}$ \\
\hline \multicolumn{3}{|l|}{ Service use } \\
\hline Testing & $\begin{array}{l}\text { Further assessment and tests as a result } \\
\text { of diagnosis (including testing of family) }\end{array}$ & $\begin{array}{l}\text { Seeking more investigations } \\
\text { Scans and imaging } \\
\text { Encourages screening of other family members at low- } \\
\text { risk of the condition }\end{array}$ \\
\hline Treatment & $\begin{array}{l}\text { Treatment and intervention as a result of } \\
\text { diagnosis }\end{array}$ & $\begin{array}{l}\text { Clear treatment path; clearer treatment protocols } \\
\text { Side-effects (of medication: sexual, agitation, suicidality, } \\
\text { emotional numbing) }\end{array}$ \\
\hline
\end{tabular}

framework (table 1) that will be used in this scoping review.

\section{Inclusion criteria}

Peer-reviewed publications including systematic or literature reviews and original studies which describe the perceived consequences for individuals labelled with a non-cancer health condition will be included. Perceived consequences can be reported from the perspectives of the individuals, their family, friends and/or carers or health professionals. As we expect individuals labelled as having a cancer condition will have different experiences to those labelled with general health conditions, studies that focus on these samples are excluded. Similarly, studies that report the consequence of labels for people using hypothetical case scenarios, or individuals with intellectual disabilities and/or social attributes such as race, sexual identity or orientation will also be excluded (see table 2 for more details).

\section{Search strategy}

A structured search, developed in collaboration with an information specialist, of five electronic databases (PubMed, Embase, PsycINFO, Cochrane, CINAHL) will be conducted to identify relevant publications. Databases will be searched from their inception. Preliminary searches were conducted in August 2019 and will be updated in June 2020. Reference lists of included articles will be searched and forward citation searching of included articles will be conducted. The full search strategy to be used is reported in the online supplemental material.

\section{Study selection}

Titles and abstracts of $10 \%$ of articles retrieved through electronic and manual searches will be independently screened by two reviewers (RS and LK) for eligibility against the pre-specified inclusion criteria. Disagreements will be resolved through discussion and consultation with additional reviewers as required. When interrater reliability $(\kappa)>0.8$ is achieved for the screened studies, remaining studies will continue to be screened by one reviewer (RS). Articles identified as unclear for inclusion will be reviewed by an additional reviewer as required.

\section{Data extraction and framework revision and validation}

Full-text publications will be obtained and the reference list reviewed. Any relevant studies found in the reference list will be screened (RS) for inclusion against the same inclusion criteria. Additional uncertainties regarding eligibility for inclusion will be resolved through discussion with other reviewers (RT or PG). Two reviewers (RS and 


\begin{tabular}{|c|c|c|}
\hline Aspect & Inclusion criteria & Exclusion criteria \\
\hline Types of studies & $\begin{array}{l}\text { Original studies (cohort, case-controlled, cross- } \\
\text { sectional, observational, Randomised Controlled Trial } \\
\text { (RCT), focus groups) } \\
\text { Synthesised studies (systematic reviews) }\end{array}$ & $\begin{array}{l}\text { Protocols (final study to be sourced) } \\
\text { Opinion pieces and commentaries } \\
\text { Quantitative cohort, case-controlled and cross- } \\
\text { sectional studies without comparator } \\
\text { Hypothetical or vignette-based studies }\end{array}$ \\
\hline Participants & $\begin{array}{l}\text { Individuals, no age limit (eg, adults, children, family, } \\
\text { carers, health professionals, general public) }\end{array}$ & Animal subjects \\
\hline Condition & $\begin{array}{l}\text { Screening and/or labelling of physical or } \\
\text { psychological health condition/s } \\
\text { Self-reported (eg, response to questions such as 'has } \\
\text { your GP ever told you that you have hypertension?') } \\
\text { Health condition confirmed (eg, medical examination } \\
\text { and testing completed as part of the study) }\end{array}$ & $\begin{array}{l}\text { Labelling of intellectual impairment, race, ethnicity, } \\
\text { sexual identity or sexual orientation } \\
\text { Labelling of cancers and cancer-related conditions } \\
\text { Self-reported conditions provided by unqualified } \\
\text { professional (eg, physiotherapist telling patient they } \\
\text { have hypertension) } \\
\text { Self-identified conditions (eg, googling of symptoms, } \\
\text { no confirmation by a medical professional) }\end{array}$ \\
\hline Outcomes & $\begin{array}{l}\text { Consequences, impact, effects of the health } \\
\text { condition label or diagnosis } \\
\text { Perceived harms and/or benefits (eg, illness burden) } \\
\text { Lived experience } \\
\text { - Psychological impact (eg, anxiety, quality of life) } \\
\text { Behaviour change (eg, participation in } \\
\text { employment) } \\
\text { - Support (eg, financial, social support) }\end{array}$ & $\begin{array}{l}\text { Effect of the health condition (eg, disease } \\
\text { mechanisms/traits) } \\
\text { Gene labelling } \\
\text { Food or nutrition labelling } \\
\text { Drug effects/effectiveness } \\
\text { Intervention effects/effectiveness } \\
\text { (eg, intervention A vs intervention B) }\end{array}$ \\
\hline Language & No language limitations & - \\
\hline Date & No date limitations & - \\
\hline
\end{tabular}

*Studies using qualitative methodologies do not require multiple group comparisons for inclusion.

ZAM) will independently extract study data from $10 \%$ of included qualitative studies and $10 \%$ of included quantitative studies using a standardised data extraction form that will be piloted prior to use. Conflicts will be resolved by a third party as required. Once interrater reliability $(\kappa)>0.8$ is achieved for extracted data, one reviewer (RS) will undertake the remaining data extraction in a staged process, with this detailed below in the extraction sections. The same staged process will be used when extracting data from quantitative and qualitative studies. Queries will be resolved through discussion with a second reviewer (ZAM).

The methods used to extract and synthesise the results of qualitative and quantitative studies are based on the meta-analytic techniques described by Sandelowski et al, ${ }^{33}$ Thomas and Harden ${ }^{34}$ and Timulak. ${ }^{35}$ Extracted data will include study characteristics (author, journal, year of publication, study country and setting), participant characteristics (number of participants, age, health condition) and quantitative or qualitative outcomes (consequences, impact, effects of the diagnostic label).

\section{Qualitative data extraction}

Data for thematic analysis will be extracted from the published study and include the authors abstracted themes and relevant, supporting quotes, reported in the primary study. Direct quotes will not be extracted in isolation to ensure data 'retains its meaning' and is not interpreted or extracted out of the context of the primary study. This qualitative meta-analysis technique has been described by Sandelowski et al, ${ }^{33}$ Thomas and Harden ${ }^{34}$ and Timulak. ${ }^{35}$

\section{Quantitative data extraction}

For studies with quantitative outcomes, extracted data will include, the text and numerical data from the results section reporting primary outcomes. ${ }^{36}$ Examples of potential quantitative measures include the Short Form Health Survey (SF-36), ${ }^{37}$ General Health Questionnaire $(\mathrm{GHQ})^{38}$ or work absenteeism.

\section{Qualitative data analysis}

The coding framework developed from social media responses will be iteratively revised using eligible studies retrieved by the electronic database search. Qualitative data will initially be extracted from a random sample of one-third of included qualitative studies and mapped to the coding framework. This framework will be expanded as additional themes emerge. The second third of included qualitative studies will be randomly selected, data extracted and mapped to the updated coding framework until data thematic saturation has been achieved. If new themes are still emerging at this point, the remaining third of qualitative studies will be analysed against the developed framework. Data saturation will be defined using indicative thematic saturation, which states data 
saturation as the non-emergence of new codes or themes that will result in expansion or revision of the coding framework. ${ }^{36}$

\section{Quantitative data analysis}

Quantitative data will be summarised narratively. ${ }^{33}$ For example, we will collate data from studies that used the SF-36, GHQ or absenteeism and summarise the findings reported in the results section. Unlike the large volume of expected qualitative studies, fewer quantitative studies with comparators are expected. Therefore, outcomes from all of the included quantitative studies will be extracted and, if possible, tabulated by condition and outcomes.

\section{Patient and public involvement}

This scoping review has no direct patient involvement.

\section{PRESENTATION OF RESULTS}

We will present study selection in a flow diagram according to PRISMA-ScR and included studies will be described in a table of characteristics. ${ }^{32}$ Results will be aggregated as appropriate. Results pertinent to the consequences of labelling of health conditions will be collated to expand those provided in table 1, with empirical data regarding the rate and severity of these consequences also examined. Additionally, a compendium of methods used to elicit the consequences of health condition labelling will be developed and methodology appraised. For quantitative studies, extracted data will be tabulated in a descriptive and/or statistical manner depending on the availability of data (ie, number of studies reporting similar outcome measures or measurement of similar constructs, such as quality of life or symptoms of anxiety) and degree of heterogeneity between studies (eg, population, clinical conditions). Should data not support a metaanalysis, results from studies which provide quantitative data will be reported in a narrative synthesis and interpreted alongside results from qualitative studies. Qualitative data will be analysed using developed frameworks (see table 1) and following established protocols for the qualitative analysis of information in the social sciences. ${ }^{39}$ The characteristics and results of all included studies will be reported in tables and summarised in text.

\section{Ethics and dissemination}

As the current study is a systematic scoping review protocol, ethics is not required. Dissemination of results will be made public via peer-reviewed publications, conference presentations and lay-person summaries on various on-line platforms (eg, The Conversation).

\section{Twitter Rae Thomas @rthomasEBP}

Acknowledgements The authors thank Justin Clark, Senior Research Information Specialist at the Institute for Evidence-Based Healthcare, Bond University for assistance with constructing the search strategy.

Contributors RS, PG and RT contributed to the conception and design of the protocol, initial public 'survey' and construction of the search terms. RS, LK and
ZAM contributed to screening and data analysis. RS, ZAM, RT and PG contributed to the drafting of the manuscript and all authors approved the final version.

Funding RS is supported by an Australian Government Research Training Program Scholarship. RT and ZAM are supported by a National Health and Medical Research Council Program grant (\#1106452). LK is supported by a Centres of Research Excellence Grant (\#1104136). PG is supported by a NHMRC Research Fellowship (\#1080042).

Disclaimer The funding sources have no role in study design, data collection, data analysis, data interpretation or writing of the report.

Competing interests None declared.

Patient consent for publication Not required.

Provenance and peer review Not commissioned; externally peer reviewed.

Supplemental material This content has been supplied by the author(s). It has not been vetted by BMJ Publishing Group Limited (BMJ) and may not have been peer-reviewed. Any opinions or recommendations discussed are solely those of the author(s) and are not endorsed by BMJ. BMJ disclaims all liability and responsibility arising from any reliance placed on the content. Where the content includes any translated material, BMJ does not warrant the accuracy and reliability of the translations (including but not limited to local regulations, clinical guidelines, terminology, drug names and drug dosages), and is not responsible for any error and/or omissions arising from translation and adaptation or otherwise.

Open access This is an open access article distributed in accordance with the Creative Commons Attribution Non Commercial (CC BY-NC 4.0) license, which permits others to distribute, remix, adapt, build upon this work non-commercially, and license their derivative works on different terms, provided the original work is properly cited, appropriate credit is given, any changes made indicated, and the use is non-commercial. See: http://creativecommons.org/licenses/by-nc/4.0/.

\section{ORCID iDs}

Rebecca Sims http://orcid.org/0000-0002-1604-8354

Luise Kazda http://orcid.org/0000-0003-4105-0402

Zoe A Michaleff http://orcid.org/0000-0002-0360-4956

Paul Glasziou http://orcid.org/0000-0001-7564-073X

Rae Thomas http://orcid.org/0000-0002-2165-5917

\section{REFERENCES}

1 Sexton $\mathrm{H}$, Heal C, Banks J, et al. Impact of new diagnostic criteria for gestational diabetes. J Obstet Gynaecol Res 2018;44:425-31.

2 Australian Bureau of Statistics. National health survey: first results, 2017-18, 2019. Available: https://www.abs.gov.au/ausstats/abs@. $\mathrm{nsf} / \mathrm{mf} / 4364.0 .55 .001$ [Accessed 30 Nov 2019].

3 Hamer M, Batty GD, Stamatakis E, et al. Hypertension awareness and psychological distress. Hypertension 2010;56:547-50.

4 Thombs BD, Kwakkenbos L, Levis AW, et al. Addressing overestimation of the prevalence of depression based on self-report screening questionnaires. CMAJ 2018;190:E44-9.

5 Noble S, Bonner C, Hersch J, et al. Could disease labelling have positive effects? an experimental study exploring the effect of the chronic fatigue syndrome label on intended social support. Patient Educ Couns 2019;102:486-93.

6 Walker MJ, Rogers WA. Diagnosis, narrative identity, and asymptomatic disease. Theor Med Bioeth 2017;38:307-21.

7 Hiremath SB, Boto J, Regnaud A, et al. Incidentalomas in spine and spinal cord imaging. Clin Neuroradiol 2019;29:191-213.

8 Doust J, Glasziou P. Is the problem that everything is a diagnosis? Aust Fam Physician 2013;42:856-9.

9 Brodersen J, Schwartz LM, Heneghan C, et al. Overdiagnosis: what it is and what it isn't. BMJ Evid Based Med 2018;23:1-3.

10 Frances A, First M, Pincus HA, et al. An introduction to DSM-IV. Hosp Community Psychiatry 1990;41:493-4.

11 Croft P, Altman DG, Deeks JJ, et al. The science of clinical practice: disease diagnosis or patient prognosis? Evidence about "what is likely to happen" should shape clinical practice. BMC Med 2015;13:20.

12 Lam DCK, Poplavskaya EV, Salkovskis PM, et al. An experimental investigation of the impact of personality disorder diagnosis on clinicians: can we see past the borderline? Behav Cogn Psychother 2016;44:361-73.

13 Allsopp K, Read J, Corcoran R, et al. Heterogeneity in psychiatric diagnostic classification. Psychiatry Res 2019;279:15-22. 
14 American Psychological Association. Diagnostic and statistical manual for mental disorders. Fifth ed. Washington, DC: American Psychiatric Association, 2013.

15 Christiansen J. Less is more: chest pain pathways in clinical care. Med J Aust 2017;207:193-4.

16 Sturm C, Witte T. [Musculoskeletal-related chest pain]. Internist 2017;58:39-46.

17 Hartvigsen J, Hancock MJ, Kongsted A, et al. What low back pain is and why we need to pay attention. Lancet 2018;391:2356-67.

18 Scherer LD, Zikmund-Fisher BJ, Fagerlin A, et al. Influence of "GERD" label on parents' decision to medicate infants. Pediatrics 2013;131:839-45.

19 Parliament of Australia. Access to real learning: the impact of policy, funding and culture on students with disability, 2016. Available: https://www.aph.gov.au/Parliamentary_Business/Committees/ Senate/Education_and_Employment/students_with_disability/Report [Accessed 30 Nov 2019].

20 Witham M. Funding the need not the label. Western Australia, Australia: AARE Conference, 2015.

21 Nickel B, Barratt A, Copp T, et al. Words do matter: a systematic review on how different terminology for the same condition influences management preferences. BMJ Open 2017;7:e014129.

22 Copp T, McCaffery K, Azizi L, et al. Influence of the disease label 'polycystic ovary syndrome' on intention to have an ultrasound and psychosocial outcomes: a randomised online study in young women. Hum Reprod 2017;32:876-84.

23 O'Keefe M. Do different diagnostic labels for low back pain influence management preferences: an online randomised controlled study. Sydney, Australia: Preventing Overdiagnosis Conference, 2019.

24 Kavookjian J, Berger BA, Grimley DM, et al. Patient decision making: strategies for diabetes diet adherence intervention. Res Social Adm Pharm 2005;1:389-407.

25 Ogden J, Branson R, Bryett A, et al. What's in a name? an experimental study of patients' views of the impact and function of a diagnosis. Fam Pract 2003;20:248-53.

26 Macdonald LA, Sackett DL, Haynes RB, et al. Labelling in hypertension: a review of the behavioural and psychological consequences. J Chronic Dis 1984;37:933-42.
27 Cotter AR, Vuong K, Mustelin L, et al. Do psychological harms result from being labelled with an unexpected diagnosis of abdominal aortic aneurysm or prostate cancer through screening? A systematic review. BMJ Open 2017;7:e017565.

28 Betsch TA, Gorodzinsky AY, Finley GA, et al. What's in a name? health care providers' perceptions of pediatric pain patients based on diagnostic labels. Clin J Pain 2017;33:694-8.

29 Lancaster AM. Impact of diagnostic versus emotional disturbance label on preservice teacher expectations of student academic, behavior, and social outcomes [PhD Thesis. Mississippi: Mississippi State University, 2016.

30 Arksey H, O'Malley L. Scoping studies: towards a methodological framework. Int J Soc Res Methodol 2005;8:19-32.

31 Peters M, Godfrey C, Mclnerney P. Joanna Briggs Institute revier's manual. The Joanna Briggs Institute, 2017.

32 Tricco AC, Lillie E, Zarin W, et al. PRISMA extension for scoping reviews (PRISMA-ScR): checklist and explanation. Ann Intern Med 2018;169:467-73.

33 Sandelowski M, Barroso J, Voils Cl. Using qualitative metasummary to synthesize qualitative and quantitative descriptive findings. Res Nurs Health 2007:30:99-111.

34 Thomas J, Harden A. Methods for the thematic synthesis of qualitative research in systematic reviews. BMC Med Res Methodol 2008;8:45.

35 Timulak L. Meta-Analysis of qualitative studies: a tool for reviewing qualitative research findings in psychotherapy. Psychother Res 2009;19:591-600.

36 Saunders B, Sim J, Kingstone T, et al. Saturation in qualitative research: exploring its conceptualization and operationalization. Qual Quant 2018;52:1893-907.

37 Ware JE, Sherbourne CD. The mos 36-item short-form health survey (SF-36). I. conceptual framework and item selection. Med Care 1992;30:473-83.

38 Goldberg D, Williams P. A user's quide to the General Health Questionnaire. Windsor, Berks: NFER-Nelson, 1988.

39 Ritchie J, Lewis J, McNaughton Nicholls C, et al. Qualitative research practice: a guide for social science students and researchers. 2nd ed. London, UK: Sage Publications Ltd, 2014. 